\title{
Use of Nanohydroxyapatite in Regenerative Therapy in Dogs Affected by Periodontopathy: Preliminary Results
}

\begin{abstract}
Background: The present study aims to analyse the in vivo behaviour of nanohydroxyapatite and to assess its regenerative capacity in dogs affected by periodontal disease.

Methods and findings: The dogs employed in the study were randomly subdivided into a control group and an experimental group. After clinical, instrumental and radiological examinations, all the subjects underwent dental prophylaxis and a bioptic sample was taken. A histopathological examination of the periodontal tissues, in correspondence to teeth with periodontopathy ranging between stages 2 and 3 , followed. Regenerative therapy with applications of nanohydroxyapatite was administered only to the dogs of the experimental group. After a period of between 35 and 40 days, a further clinical, instrumental and radiological examination was carried out and a bioptic sample taken solely on the dogs whose histological examinations showed changes ascribable to periodontal disease. The results of the histopathological examination demonstrated that only the dogs in the experimental group, who underwent dental prophylaxis together with the administration of nanohydroxyapatite, showed clear signs of improvement with respect to their initial condition.
\end{abstract}

Conclusions: In conclusion the study demonstrated that the nanohydroxyapatite represents a valid osteoconductive and osteoinductive graft product and confirmed its regenerative potential in periodontal therapy in dogs.

Keywords: Periodontal disease, Dog, Nanohydroxyapatite, Regenerative therapy, Histopathological examination

\author{
Cecilia Vullo', \\ Marina Meligrana², \\ Giacomo Rossi ${ }^{2}$, \\ Adolfo M. Tambella², \\ Fabrizio Dini², \\ Angela Palumbo \\ Piccionello² \\ Andrea Spaterna ${ }^{2}$
}

1 University of Camerino, School of Pharmacology, Via Madonna delle Carceri, 62032 Camerino (MC), Italy

2 University of Camerino, School of Biosciences and Veterinary Medicine, Via Circonvallazione 93/95, 62024 Matelica (MC), Italy

\section{Corresponding author: Marina Meligrana}

” marina.meligrana@hotmail.it

University of Camerino, School of Biosciences and Veterinary Medicine, Via Circonvallazione 93/95, 62024 Matelica (MC), Italy

Tel: +390737403447

\section{Introduction}

The most common disorders found during clinical examinations in dogs in all age ranges are represented by oral diseases and, amongst these, Periodontal Disease (PD) is the most frequently diagnosed [1]. It is a multifactorial infection which attacks the periodontium, and bacterial plaque is the most important etiological factor. PD can have numerous complications, both local (oro-nasal fistulas, pulpitis, pathological fractures, osteomyelitis) and systemic (renal, cardiac, lung and liver diseases, diabetes mellitus, negative effects on pregnancy) [2].

Guided Tissue Regeneration (GTR) is one of the latest techniques introduced for regenerative purposes in the control of this disease. It aims to rebuild tissues compromised by PD, restoring them to their original condition by utilizing barrier membranes, bone grafts and bioactive materials which promote the neoformation of the desired tissues [3].

There has been a recent growth in interest in nanotechnology in many fields and, in particular, in dentistry significant applications ofnumerous biomaterials are being developed, above all in the regeneration of periodontal tissues. Among the alloplastic graft materials, research into the use hydroxyapatite $\left[\mathrm{Ca}_{10}\left(\mathrm{PO}_{4}\right)_{6}(\mathrm{OH})_{2}\right]$ in the medical field has long been carried out. Being a major component of the inorganic mineral part of bone and teeth, it presents a tested biocompatibility [4-11]. Its use in oral and maxillofacial surgery, periodontology, orthopaedics and vertebral surgery has been widely documented [12-15]. It behaves as an osteoconductive material, which acts as a scaffold for the new bone growth. Hydroxyapatite is used mainly to fill periodontal 
defects, and the possibility of obtaining the nanocrystalline form has further improved its penetration capacity also conferring osteoinductive properties. Several studies have shown that, given its chemical and chrystallographic affinity with the inorganic components of bone, this product is able to set up chemical links and to guarantee a more rapid integration of the implants with the bone and the surrounding tissues [16-18]. Another interesting element is that the nanohydroxyapatite proved to be less phlogogenic than the standard hydroxyapatite and to induce a higher production of alkaline phosphatase and osteocalcin, indicating a greater bone production [19-21].

The aim of this work has been to analyse the in vivo behaviour of nanohydroxyapatite (NIA) and to confirm its regenerative capacity in dogs with periodontal disease stages 2 and 3 by means of our clinical and experimental contribution. A new apatite - Neo Active Apatite ${ }^{\circledR}$ - with crystals varying in size between 70 and 100 $\mathrm{nm}$ and developed by an Italian firm (Ghimas from Casalecchio di Reno, Bologna) was utilized.

\section{Materials and Methods}

Ethical approval for the study was obtained from the Italian Ministry of Education, University and Research. For the purposes of this study, 40 mixed-breed dogs with periodontal disease, ranging from between 5 and 15 years old and weighing between 15 and $40 \mathrm{~kg}$, were selected. PD varied between stages 2 and 3 . The classification is in accordance with the AVDC periodontal disease classification. The dogs were randomly subdivided into two homogenous groups: GROUP C (control group) and GROUP N (experimental group). All the subjects underwent a generalphysical examination, a physical examination of the oral cavity, a blood test and a cardiological examination. Dogs with clearly malignant proliferative lesions and cardiovascular or metabolic complications where surgical and anaesthetic procedures were contraindicated were excluded from the study.

A thorough clinical and instrumental intraoral examination of the periodontium was carried out under general anaesthesia, followed by a radiological examination in order to make an accurate assessment of the stage of the disease. The instrumental examination was performed utilizing a Williams graduated probe, which was run along the gingival sulcus of each tooth in order to highlight the presence of subgingival pockets, gingival recession or attachment loss (Figure 1). A dental explorer was utilized to spot possible loss of substanceof the mineralized components of each single tooth. All clinical signsobserved were registered onto a dental chart and photographed.

The radiological examinations were carried out using a standard digital machine (FUJIFILM FCR CAPSULA). Right and left oblique latero-lateral extraoral and dorso-ventral and ventro-dorsal intraoral projections were taken without intubation. The extraoral projections allowed for radiographic visualization of the mandibular and maxillary arches without superimposition, since the images were taken with the mouth wide open at an inclination of $30^{\circ}$ with respect to the orthogonal plane, utilizing a foam rubber support (Figure 2). The intraoral shots, with the film inserted inside the mouth, allowed radiographic visualization of the rostral part of the maxilla and of the mandible, including the canines (dorso-ventral and ventro-dorsal views) (Figure 3). To avoid excessive lengthening of the roots of the canines in both arches, the intraoral shots were taken using the bisectingangle technique, inclining the radiographic beam perpendicular to the bisector of the angle formed by the long axis of the tooth and of the radiographic cassette.

Once intubation was performed, each patient was taken into the operating theatre, kept under inhalationanaesthesia and underwent dental treatment. The subjects belonging to the GROUP $N$ also underwent guided regenerative periodontal therapy. The dental prophylaxis, respecting the norms of disinfection and asepsis as far as possible, was carried out by means of tartar ablation,gingival curettage (to remove the infected soft tissue lining of the periodontal pockets), rinsing the gingival sulcus with $0.12 \%$ chlorexidine and, finally, polishing the enamel. The teeth where both the instrumental and radiographic examinations highlighted furcation exposure and over $50 \%$ bone

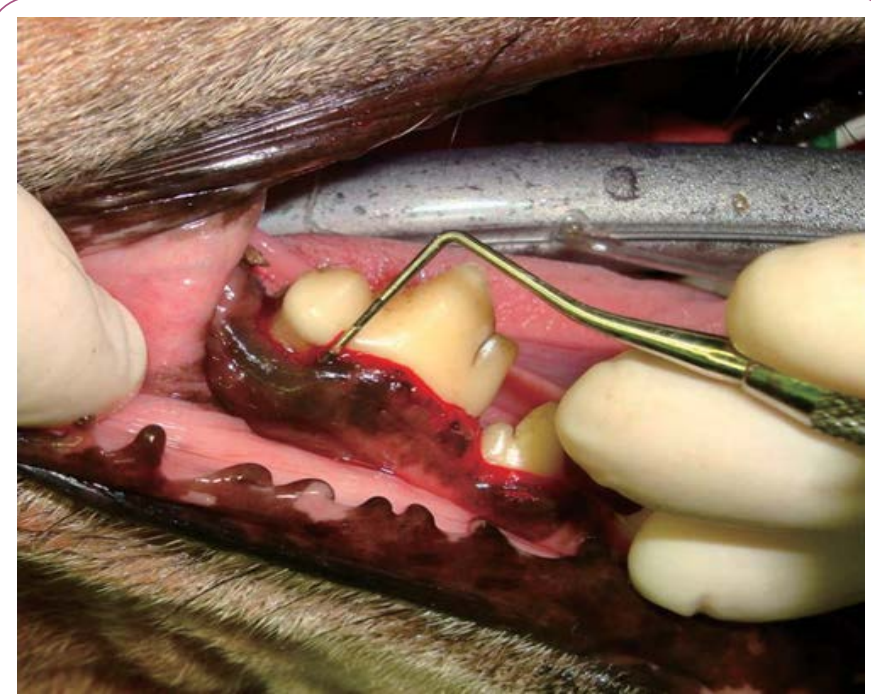

Figure 1 Instrumental examination utilizing a graduated periodontal probe.

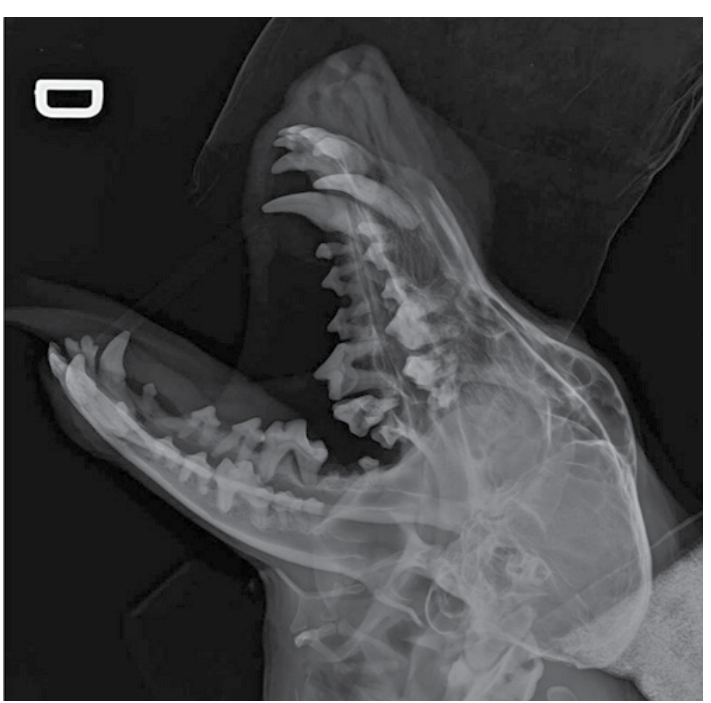

Figure 2 Right oblique latero-lateral extraoral projection. 


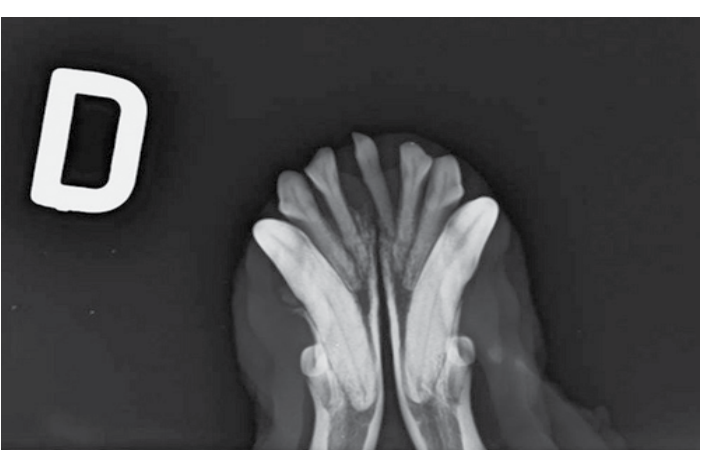

Figure 3 Ventro-dorsal intraoral projection.

resorption (PD stage 4 - advanced periodontitis), were extracted. One tooth with a degree of periodontopathy varying between stages 2 (early periodontitis) and 3 (moderate periodontitis) was selected and a bioptic sample of the periodontal tissues of the bone and gingiva from every single dog in each group was taken.

In order to carry out the biopsy it was necessary to create a full thickness (mucoperiosteal) nondisplaced flap. Firstly, a crevicular horizontal incision was performed using a number 15 scalpel blade and a second vertical releasing incision was created mesial or distal to the target tooth, thus obtaining a triangular flap. With the aid of a sharp Molt periosteal elevator, the flap was elevated from the alveolar bone respecting the margins as far as possible (Figure 4). The exposed surface of the root was planed using a Gracey curette, after the removal of the infected granulation tissue. Subsequently, the biopsy was performed using Rongeur bone cutting forceps (Figure 5) and the fragment of tissue was immersed in $10 \%$ neutral buffered formalin solution and underwent histopathological examination. After the biopsy, only the subjects belonging to the GROUP N were treated with the guided regenerative periodontal therapy by means of an application of Neo Active Apatite Powder ${ }^{\circledR}$, utilizing a Williger bone spoon (Figure 6). The grains of powder were dampened with saline solution to improve adherence. The flaps were then repositioned to their original sites and sutured with 4-0 absorbable monofilament suture material on a reverse cutting needle (Monocryl, Ethicon ${ }^{\circledR}$ ) (Figure 7).

The dogs were then brought roundand, finally, discharged after one day of hospitalization.

After a period of between 35 and 40 days, only to the dogs where the histological examination of the biopted tissue had highlighted lymphocyte and plasmacellular infiltrations with abundant active osteoclasts and macrophages, together with bone resorption phenomena, were re-examined. The dogs whose histological examination did not highlight any changes attributable to periodontal disease (13 in total), were excluded from the reexamination. The 27 remaining dogs who were re-examined, were given,under general anaesthesia, asecond clinical, instrumental, radiographic and histological examinationonly in relation to the areas wherethe previous biopsy was carried out.

In total, 12 dogs belonging to GROUP C and 15 to GROUP N were re-examined.

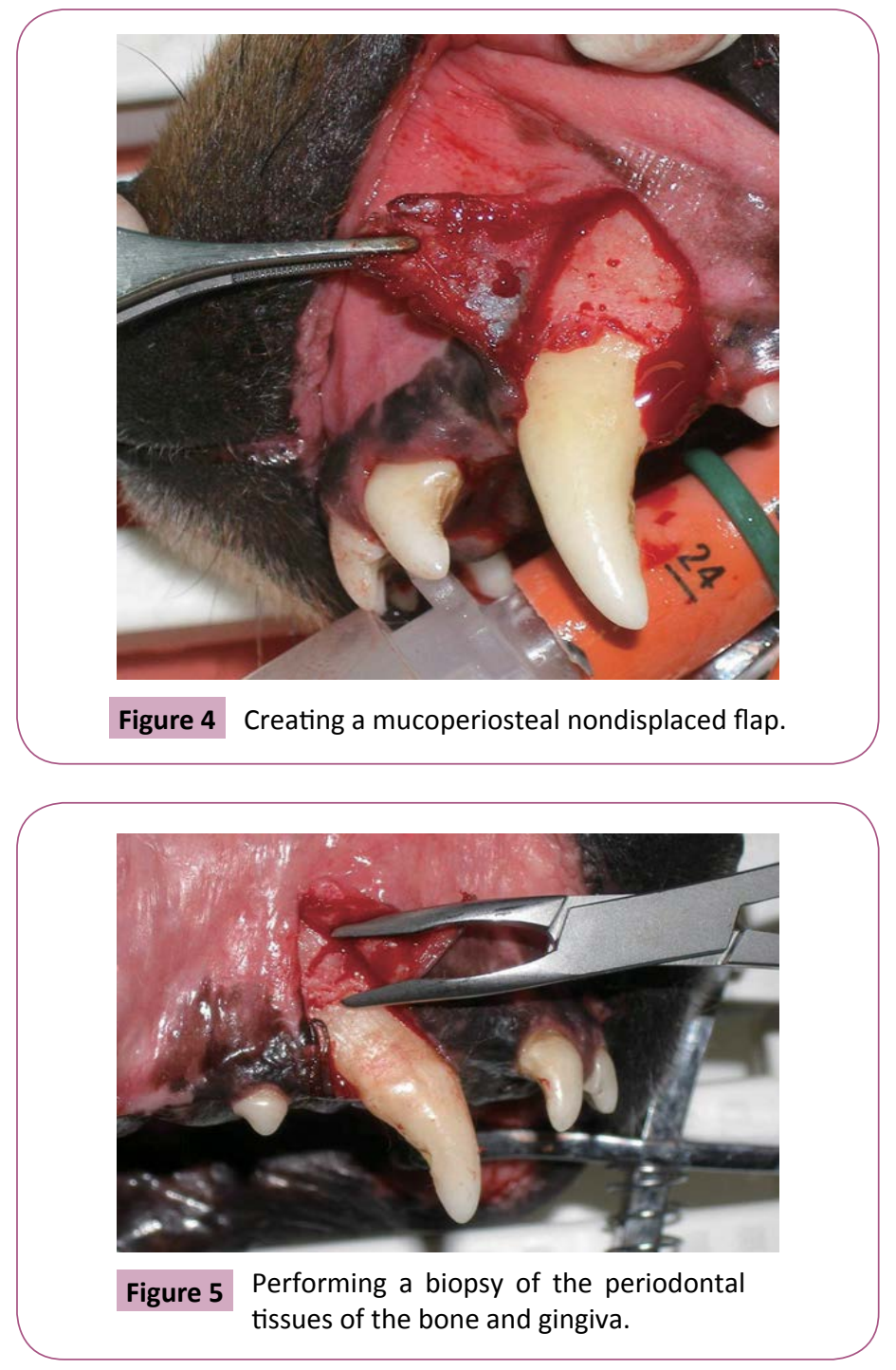

\section{Results}

No signs of rejection or dehiscence of the mucoperiosteal flap were registered in any of the dogs who underwent the re-examination. The clinical assessment and instrumental examinations of the 12 subjects appertaining to GROUP C highlighted a slight to moderate gingival inflammation in 9 of the dogs, characterized by oedema and rednessof the free margin, while in 3 subjects there were no appreciable phenomena of inflammation or signs of spontaneous bleeding. Probing with the use of a Williams graduated probe showed the level of attachment loss to be superimposableto the initial values. The $x$-ray image of the area where the biopsy was taken did not highlight areas of bone rarefaction greater than those shown on the initial images. In 5 subjects, the histological examination confirmed the same degenerative phenomena as in the first sample, characterized by osteoclastic/osteolithic processes attributable to signs of periodontitis; in 5 dogs, there was a worseningof the degenerative process, either with areas of exuberant or hyperplastic granulation tissue infiltrated by numerous polymorphonucleated cells, or with tissue inflammation starting from the periosteum, taking over the paraosteal area and extending to the gingiva, with widespread dystrophic and 


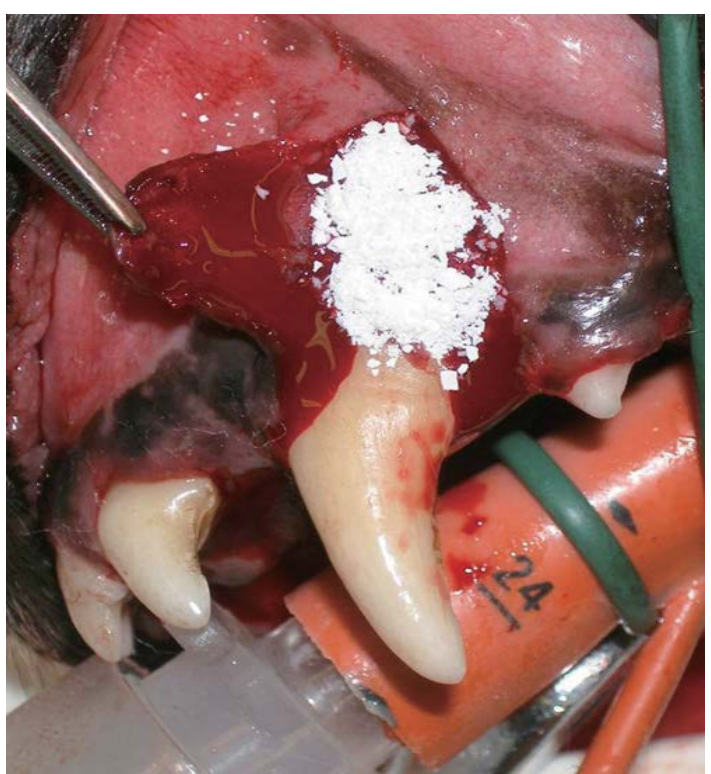

Figure 6 Application of Neo Active Apatite Powder $^{\circledR}$.

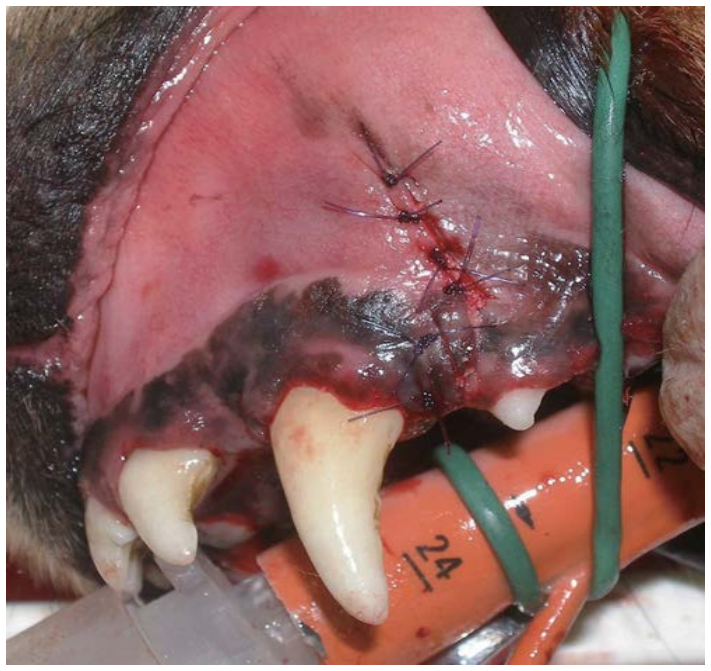

Figure 7 Repositioning the flap.

heterotopic calcification and no proliferative phenomena. In 2 cases there proved to be widespread infiltration and erosion of the gingival epithelium, with an extensive dysplastic aspect of the basal layer, while there were clear signs of alveolar bone resorptionand irregularity, with multiple necrotic microfoci.

In 4 of the 15 subjects belonging to the GROUP N, clinical assessment and instrumental examinations highlighted a slight degree of gingival inflammation, characterized by oedema and redness of the free gingival margin with no spontaneous bleeding, while in 11 subjects there were no clinically appreciable inflammatory phenomena. The examination carried out using a Williams probe, showeda regression in the levels of attachment loss in all the dogswith respect to the initial examinations, which proved to be total in 8 of the subjects and partial in 7 . The radiographic examination of the area of the biopsy did not highlight any phenomena of bone rarefaction, nor any osteoproduction greater than that shown in the initial images. In 5 of the animals belonging to this group, where the results of the initial histological examinations highlighted a mononuclear lymphoplasmacytic cell infiltration of the mucosal corium, ascribable to phenomena of chronic gingivitis, the second sample showed the gingiva to have a normal structure with absence of inflammatory cell infiltration. In 10 subjects, the initial histopathological report highlighted a situation ascribable to multifocal necrotic phenomena, with areas of periostitis-osteomyelitis, characterized by severely altered appearance of the bone and widespread osteoclastic resorption (Figure 8); in 8 of these subjects, the result of the second sample was characterized by osteoblast repopulating together with proliferative-reparative periosteal processes, with absence of inflammatory cell infiltrate, while in 2 subjects the alveolar bone had a paraphysiological appearance (Figure 9).

\section{Discussion}

Although over the last forty years there has been significant progress towards the understanding of the ethiopathogenesis of PD, traditional methods for formulating a clinical diagnosis have remained fundamentally unchanged:nowadays, the diagnostic and staging evaluation for PD is traditionally based on clinical, instrumental and radiographic parameters which, lacking in scientific rigour, prove to be insufficient even from a prognostic point of view. In the first place, in fact, clinical assessment - such as probing depth and attachment loss - are subjective in character; furthermore, the results obtained by means of the above parameters are tied to the moment in which the examination takes place and, thus, will vary in relationship to temporary and specific conditions like, for example, the inflammatory condition. Studies carried out on the progression of PD have shown that it is a pathological condition in evolution, which alternates between periods of active tissue destruction and periods of involution or dormancy [22,23]. Hence, traditional clinical assessment should not be restricted to a single periodontal examination [24].

As reported in various studies, a radiographic examination underestimates the alveolar bone level, both from the point of view of resorption - in as much as it only highlights bone loss when it exceeds $30-40 \%$ - and also in a regenerative sense, because a minimum degree of mineralization must be present in order for it to be radiographically visualized [25].

Considering the numerous variables which can influence the evaluation of the stage of PD, a biopsy of the periodontal tissues represents an additional fundamental examination which is essential for a correct diagnosis [26]. In fact, although the biopsy - in particular that of the bone - is a technique which potentially has some contraindications in areas affected by resorption, it remains the only way to determine exactly which inflammatory elements are involved and the gravity of the alveolar resorption. The histopathological examination, therefore, represents the gold standard both for the clinical assessment of the disease and the formulation of a prognosis.

Thus, to establish the outcome of a periodontal regenerative therapy, it is necessary to carry out both the clinical, instrumental and radiographic examinations, as well as a histopathological analysis, while, at the same time, taking into consideration the above-mentioned limitations.

In particular, in this work, the criteria whereby the subjects were enlisted and, subsequently, the method by which the PD stage 


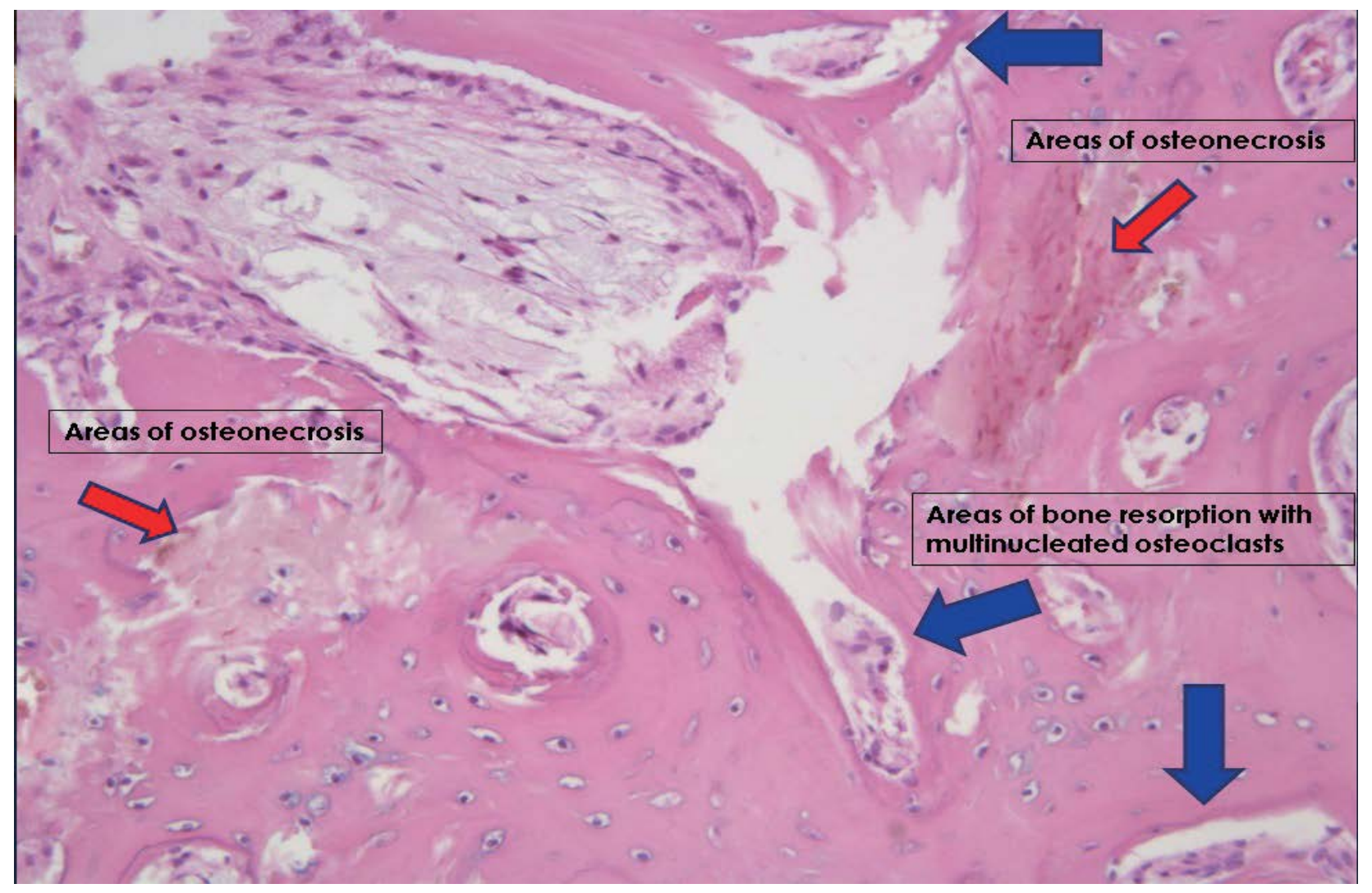

Figure 8 Histopathological image before treatment.

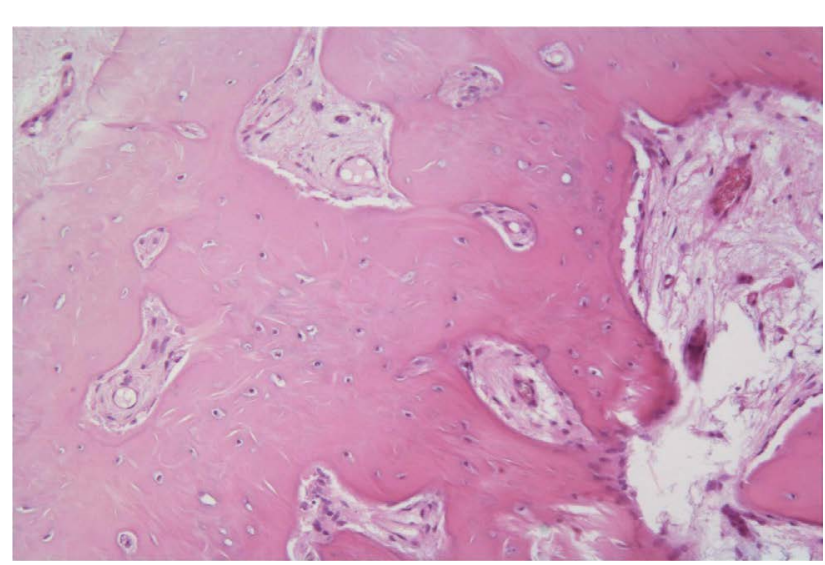

Figure 9 Histopathological image after treatment.

was assessed, were based on traditional systems. Having carried out a guided periodontal regenerative therapy, we felt that it was essential to take a sample of a fragment of periodontal tissue, taking advantage of the histopathological examination in order to evaluate the effectiveness of NIA as a graft material. On the basis of the results obtained, it is abundantly clear that the histopathological condition of all the subjects belonging to GROUP C - who underwent exclusively professional periodontal treatment - showed no signs of improvement. In fact, the results proved to be analogous in 5 out of 12 of the subjects and decidedly worsened in the remaining 7 . On the other hand, the histological examination of all the dogs belonging to GROUP $\mathrm{N}$-to which the professional periodontal treatment had been combined with the application of NIA -showed a marked improvement with respect to the initial condition. The structure of the mucosal coriumappeared normal and showed osteoblast repopulating. Furthermore, in 2 cases the bone had a paraphysiological appearance.

\section{Conclusion}

we can affirm that the utilization of NIA as a graft material in regenerative periodontal therapy has led to very satisfactory results which, given the limitations of the diagnostic instruments available, we felt should be confirmed unquestionably by means of a histopathological examination. We, therefore, believe - in accordance with the results obtained in human medicine $[27,28]$ - that NIA constitutes both a valid osteoconductive as well as osteoinductive graft product in dogs.

We feel it necessary to point out that the results obtained appear even more encouraging, given the fact that the study was carried out on dogs from the kennel, where regular daily treatment had not been possible. In fact, the animals in question had been deprived of the considerablefurther benefits that the daily care adds to the dental prophylaxis and to regeneration therapies. We consider it essential to widen the number of subjects enlisted in the study and to improve the results obtained, both by utilizing dogs whose owners are willing to carry out the above-mentioned treatment and by further standardizing diagnostic assessment methods. 


\section{References}

1 Niemiec BA (2008) Periodontal therapy. Top Companion Anim Med 23(2): 81-90.

2 Niemiec BA (2013) Veterinary Periodontology. Oxford, UK: WileyBlackwell.

3 Lommer MJ, Wiggs RB, Anderson JG (2012) Osteoconductive and osteoinductive agents in periodontal surgery. In: Verstaete FJM, Lommer MJ (eds). Oral and Maxillofacial Surgery in Dogs and Cats. 1st ed: Sauders185-192.

4 Kent JN (1986) Reconstruction of the alveolar ridge with hydroxyapatite. Dent Clin North Am 30: 231-257.

5 Kent JN, Zide MF, Kay JF, Jarcho M (1986) Hydroxylapatite blocks and particles as bone graft substitutes in orthognathic and reconstructive surgery. J Oral Maxillofac Surg 44: 597-605.

6 Orly I, Kerebel B, Abjean J, Heughebaert M, Barbieux I (1989) Hydroxyapatite biomaterial implanted in human periodontal defects: an histological and ultrastructural study. Bull Group Int Rech Sci Stomatol Odontol 32: 79-86.

7 Weinlaender M (1991) Bone growth around dental implants. Dent Clin North Am 35: 585-601.

8 Bagambisa FB, Joos U, Schilli W (1993) Mechanisms and structure of the bond between bone and hydroxyapatite ceramics. J Biomed Mater Res 27: 1047-1055.

9 Sempuku T, Ohgushi H, Okumura M, Tamai S (1996) Osteogenic potential of allogeneic rat marrow cells in porous hydroxyapatite ceramics: a histological study. J Orthop Res 14: 907-913.

10 Okumura M, Ohgushi H, Dohi Y, Katuda T, Tamai S, et al. (1997) Osteoblastic phenotype expression on the surface of hydroxyapatite ceramics. J Biomed Mater Res 37: 122-129.

11 Inoue K, Ohgushi H, Yoshikawa T, Okumura M, Sempuku T, et al. (1997) The effect of aging on bone formation in porous hydroxyapatite: biochemical and histological analysis. J Bone Miner Res 12: 989-994.

12 Shepperd JA, Apthorp H (2005) A contemporary snapshot of the use of hydroxyapatite coating in orthopaedic surgery. J Bone Joint Surg Br 87: 1046-1049.

13 Oshima M, Matsuzaki H, Tokuhashi Y, Okawa A (2010) Evaluation of biomechanical and histological features of vertebrae following vertebroplasty using hydroxyapatite blocks. Orthopedics 33: 89-93.

14 Hahn BD, Park DS, Choi JJ, Ryu J, Yoon WH, et al. (2013) Osteoconductive hydroxyapatite coated PEEK for spinal fusion surgery. Appl Surf Sci 283: 6-11.

15 Durmuslar MC, Alpaslan C, Alpaslan G, Cakır M (2014) Clinical and radiographic evaluation of the efficacy of platelet-rich plasma combined with hydroxyapatite bone graft substitutes in the treatment of intra-bony defects in maxillofacial region. Acta Odontol Scand 72: 948-953.

16 Ashammakhi N, Ndreu A, Yang Y, Ylikauppila H, Nikkola L, et al. (2007) Tissue engineering: a new take-off using nanofiber-based scaffolds. J Craniofac Surg 18: 3-17.

17 Kasaj A, Willershausen B, Reichert C, Röhrig B, Smeets R, et al. (2008) Ability of nanocrystalline hydroxyapatite paste to promote human periodontal ligament cell proliferation. J Oral Sci 50: 279285.

18 Ozak ST, Ozkan P (2013) Nanotechnology and dentistry. Eur J Dent 7: 145-151.

19 Huang J, Best SM, Bonfield W, Brooks RA, Rushton N, et al. (2004) In vitro assessment of the biological response to nano-sized hydroxyapatite. J Mater Sci Mater Med 15: 441-445.

20 Dhivya S, Saravanan S, Sastry TP, Selvamurugan N (2015) Nanohydroxyapatite-reinforced chitosan composite hydrogel for bone tissue repair in vitro and in vivo. J Nanobiotechnology 13: 40.

21 Salgado CL, Grenho L, Fernandes MH, Colaço BJ, Monteiro FJ (2015) Biodegradation, biocompatibility and Osteoconduction Evaluation of Collagen-NanoHydroxyapatite Cryogels for Bone Tissue Regeneration. J Biomed Mater Res A.

22 Goodson JM, Tanner AC, Haffajee AD, Sornberger GC, Socransky SS (1982) Patterns of progression and regression of advanced destructive periodontal disease. J Clin Priodontol 9: 472-481.

23 Socransky SS, Haffajee AD, Goodson JM, Lindhe J (1984) New concepts of a destructive periodontal disease. J Clin Periodontol 11: 21-32.

24 Wolf DL, Lamster IB (2011) Contemporary concepts in the diagnosis of periodontal disease. Dent Clin North Am 55: 47-61.

25 Verstraete FJM (1998) Self-Assessment Colour Review of Veterinary Dentistry. London: Manson Publishing.

26 Strietzel FP, Reichart PA, Graf HL (2007) Lateral alveolar ridge augmentation using a synthetic nano-crystalline hydroxyapatite bone substitution material (Ostim): preliminary clinical and histological results. Clin Oral Implants Res 18: 743-751.

27 Al Machot E, Hoffmann T, Lorenz K, Khalili I, Noack B (2014) Clinical Outcomes after Treatment of Periodontal Intrabony Defects with Nanocrystalline Hydroxyapatite (Ostim) or Enamel Matrix Derivatives (Emdogain): A Randomized Controlled Clinical Trial. Biomed Res Int Epub.

28 Kasaj A, Rohrig B, Zafiropoulos GG, Willershausen B (2008) Clinical evaluation of nanocrystalline hydroxyapatite paste in the treatment of human periodontal bony defects: a randomized controlled clinical trial: 6-month results. J Periodontol 79: 394-400. 